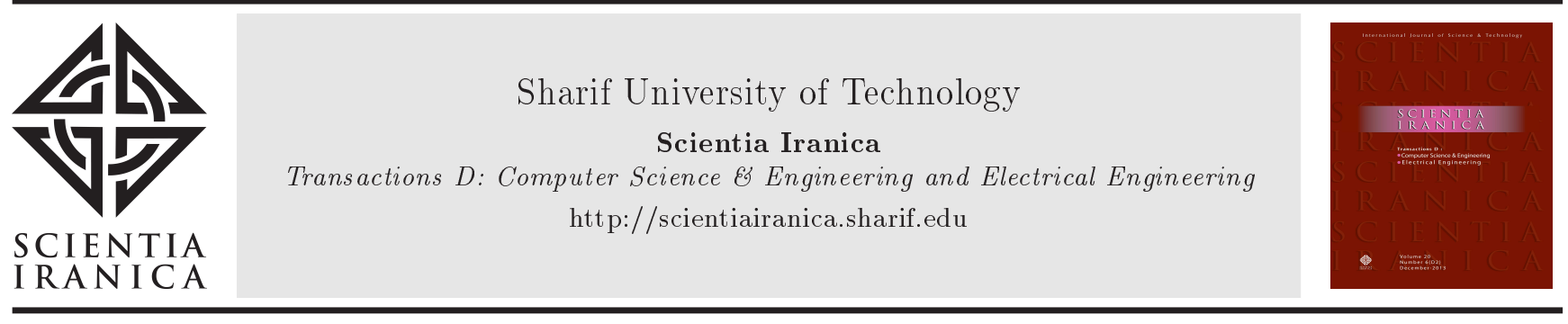

\title{
Adaptive radar signal detection in autoregressive interference using Kalman-based filters
}

\author{
M. Dorostgan ${ }^{\mathrm{a}}$ and M.R. Taban ${ }^{\mathrm{b}, \mathrm{a}, *}$ \\ a. Department of Electrical Engineering, Yazd University, Yazd, P.O. Box 89195-741, Iran. \\ b. Department of Electrical and Computer Engineering, Isfahan University of Technology, Isfahan, P. O. Box 84156-83111, Iran.
}

Received 6 January 2018; received in revised form 17 April 2019; accepted 17 June 2019

\section{KEYWORDS \\ Radar; \\ Adaptive detection; \\ Autoregressive \\ interference; \\ Primary data; \\ Kalman filter.}

\begin{abstract}
The present study deals with adaptive detection of radar target signal with an unknown amplitude embedded in Gaussian interference that has been modeled as an AR process. Application of such a model to the interference decreased the number of parameters to be estimated; therefore, less or even no secondary data were required to obtain a detector with the desired performance. Herein, detection was accomplished based on only the primary data. The authors resorting to the modern Kalman filtering technique developed the conventional GLRT-based detection in the presence of AR interference and proposed two new detectors: AREKF based on extended Kalman filter and ARUKF based on unscented Kalman filter. The performance assessment conducted by Monte Carlo simulation compared the proposed detectors with the existing ones based on the generalised likelihood ratio test and Kalman filter. The results revealed that the ARUKF detector could significantly outperform other detectors in terms of detection for both small number of primary datasets and high Signal-to-Noise Ratio (SNR).
\end{abstract}

(C) 2021 Sharif University of Technology. All rights reserved.

\section{Introduction}

The present study considers a coherent pulsed radar that transmits a train of pulses to detect targets around the radar. After pre-processing and sampling the received signal in the receiver, a data vector is formed that comprises $N$ successive returned samples from range Cell Under Test (CUT). One of the most important challenges while dealing with the problem of radar target detection is the lack of accurate information of interference (clutter plus noise). Even if we suppose that the interference has Gaussian model, there is usually not enough information about the

\footnotetext{
*. Corresponding author.

E-mail addresses: mdorostgan@gmail.com (M. Dorostgan); mrtaban@cc.iut.ac.ir (M.R.Taban)
}

doi: $10.24200 /$ sci.2019.50136.1534 covariance matrix of interference; hence, optimum Neyman-Pearson detector is not implementable.

A conventional method for tackling the mentioned problem is to use Generalised-Likelihood-Ratio-Test (GLRT) based detectors such as Kelly [1], Adaptive Matched Filter (AMF) [2], and two-dimensional detector [3] in which the covariance matrix of interference is estimated through Maximum Likelihood (ML) approach. In these and similar types of detectors, the covariance matrix is generally estimated from a number of independent and identically distributed sample vectors achieved through receiving data from the range cells adjacent to the CUT. For appropriate estimation, these samples, often called training or secondary data, must contain only the same interferences as the CUT. Of note, the accuracy of the ML estimation relies on the amount of data used for estimation. For good and reliable estimation, $K>2 N$ secondary data vectors are required [4]. Unfortunately, in some applications 
such as airborne radar, providing a sufficient number of secondary data vectors to achieve a detector with satisfactory performance may be impractical due to clutter heterogeneity or outliers [5].

Some solutions have been already addressed in the literature with less or even no need for secondary data for target detection such as resort to covariance matrix estimation through the Bayesian approach [6]. Another approach is to apply the structural information (in addition to the Hermitian property) about the covariance matrix such as circulant structure [7], Toeplitz property [8], and especially Auto-Regressive (AR) model $[9,10]$. An interesting idea was addressed in this study: assuming the AR model for interference is equivalent to considering a model with a specific analytical structure for the interference covariance matrix. Fortunately, for the interference modeled as an AR process, the model order is rather low in terms of practical applications of radar and active sonar ranging from two to five in the former applications, and it has been chosen up to eight in the latter applications [11]. To find a detector with the desired performance, the AR model for the interference decreases the number of parameters to be estimated; therefore, less or even no secondary data was needed.

In recent years, using the clutter with the AR model has received substantial attention in the literature. In [9], Kay presented a GLRT-based adaptive detector using an AR model for the interference with the assumption that the target signal is completely known. In [12], a GLR detector of radar target with an unknown amplitude embedded in the interference with AR model was introduced in which only the primary data from a pulsed radar with a single antenna was used. This detector was developed using the secondary data in [13], a multichannel radar in [14], and a MultiInput Multi-Output (MIMO) radar in [15]. In [16], the problem of detecting the range-distributed targets in the presence of structured interference modeled as an AR Gaussian process was investigated with no secondary data and therein, both the clutter homogeneity and heterogeneity in the cells under test were taken into account. Given the interference with the AR model in [17], the model coefficients were first estimated using some methods such as Yule-Walker. Then, the inverse covariance matrix of the interference was obtained by those coefficients, and AR-modelbased adaptive detection was performed by GLRT detector. In [18], the GLRT-based detection of a moving target embedded in non-homogeneous environments with distributed MIMO radars was considered, and the interference was modeled as an AR process. Moving Target Detection (MTD) was extended in [19] to the distributed MIMO radar on moving platforms with the consideration of the effects of platform motion and estimation of the order of AR process. In [20], the
AR spectral estimation and its application on weak target detection were investigated based on the fractal properties of sea clutter in the power spectrum domain regarded as an AR process. The multichannel AR model for the interference was used in Space-Time Adaptive Processing (STAP) for phased array radar applications. This model was employed in $[21,22]$ to propose the parametric detectors containing AMF, Rao, and GLRT.

According to the aforementioned detectors, modeling the interference as an AR process is conventional and acceptable in adaptive radar target detection. In this category of detectors, unknown parameters are usually estimated through the ML approach. Although the ML estimation is featured by useful properties such as asymptotically efficiency and consistency, its accuracy depends on the length of the data set which is operationally finite and inadequate. Therefore, it may be possible to achieve better results using optimal filtering techniques. The linear relationship between the received signal and interference as well as recursive form of the interference modeled as an AR process was the incentive to form a Gauss-Markov model and estimate the AR model coefficients by Kalman Filter $(\mathrm{KF})$. Although the KF is widely applied in some fields such as tracking [23, and references therein], and [24,25] it has received little attention in the field of radar target detection. This study later reveals that use of $\mathrm{KF}$ in the estimation procedure will significantly improve the detection performance, especially in case of no secondary data and limited primary data. Another advantage of $\mathrm{KF}$ in online radar detection is that due to the sequential processing of the estimator, if the estimation operation stops during its execution (for any reason such as expiring the processing time), the result of processing will not be corrupted and an estimation of the intended parameters is available.

In this study, based on the modern Kalman filtering technique, the conventional GLRT-based detection was developed in the presence of AR interference. To this end, a coherent pulsed radar with a single antenna was employed that used $N$ successive returned samples from the CUT for adaptive detection of a likely slow fluctuating target embedded in the interference modeled as an AR complex Gaussian process. Herein, the detection is performed with no secondary data, and the unknown parameters contain the complex amplitude of the target signal as well as the variance of the white noise and coefficients of the AR model. In the detector proposed in [26], the AR model coefficients are estimated using the KF. The problem of this method, which will be further discussed in the next section, is that the observation equation in Gauss-Markov model is naturally nonlinear which is approximated to a linear equation by substituting some state variables from the previous stage. To overcome this problem in this study, 
the Extended KF (EKF) and Unscented KF (UKF) were employed, indicating that these methods outperformed those presented in $[12,26]$. In addition, the detector based on the UKF significantly outperformed others in terms of detection for the small number of primary data sets and high Signal-to-Noise Ratio (SNR).

The rest of this paper is organized as follows. The next section is dedicated to modeling the signal and interference and a brief review of conventional GLRTbased detection in the presence of AR interference. Section 3 addresses the estimation of the AR model coefficients using the KF and proposed detectors based on EKF and UKF. Section 4 evaluates the performance of the proposed detectors using Monte Carlo simulation and compares it with other detectors. Finally, Section 5 concludes this study.

\section{Problem statement and GLRT-based detection}

Consider a coherent pulsed radar with a single antenna that transmits a train of pulses. The receiver forms a primary data vector containing $N$ successive returned samples from the CUT in one Coherent Processing Interval (CPI) for adaptive target detection in that CUT. Hence, a binary hypothesis test problem shows up:

$$
\begin{cases}\mathrm{H}_{0}: & \mathbf{y}=\mathbf{n} \\ \mathrm{H}_{1}: & \mathbf{y}=\mathbf{n}+\alpha \mathbf{s}\end{cases}
$$

where $\mathbf{y}=\left[\begin{array}{llll}y_{1} & y_{2} & \cdots & y_{N}\end{array}\right]^{T} \in \mathbb{C}^{N \times 1}$ is the data vector received from the CUT with $T$ denoting the transpose operator. This vector under $\mathrm{H}_{0}$ contains only the interference vector $\mathbf{n}=\left[\begin{array}{llll}n_{1} & n_{2} & \cdots & n_{N}\end{array}\right]^{T} \in$ $\mathbb{C}^{N \times 1}$ and includes the target vector as well as the interference under $\mathrm{H}_{1}$. The target is modeled as $\alpha \mathbf{s} \in \mathbb{C}^{N \times 1}$, where $\alpha$ is a complex amplitude assumed to be deterministic and unknown, and $\mathbf{s}=$ $\left[\begin{array}{lllll}1 & e^{j \theta} & e^{j 2 \theta} & \cdots & e^{j(N-1) \theta}\end{array}\right]^{T} \in \mathbb{C}^{N \times 1}$ is a known vector corresponding to the known Doppler shift of target. On the radars, the known Doppler shift assumption is common [1,4,27], mainly due to the use of filter banks in the classical radars and extraction of the signal spectrum using Fast Fourier Transform (FFT) in the digital radars based on new signal processing techniques. The interference assumed to be an AR complex Gaussian process with a known model order $M(M<N)$ is obtained from:

$$
n_{n}=\sum_{j=1}^{M} a_{j} n_{n-j}+w_{n} .
$$

The interference vector is represented by $\mathbf{n} \sim$ $A R N\left(\mathbf{a}, \sigma^{2}, M\right)$, where both of the coefficient vector $\mathbf{a}=\left[\begin{array}{llll}a_{1} & a_{2} & \cdots & a_{M}\end{array}\right]^{T}$ and variance $\sigma^{2}$ belonging to the complex white Gaussian noise $w_{n}$ are deterministic and unknown. The Probability Density Function (PDF) of the received vector under two hypotheses $\mathrm{H}_{0}$ and $\mathrm{H}_{1}$ and the ratio of likelihood functions can be written as follows [12]:

$$
\begin{aligned}
& f_{y}\left(\mathbf{y} \mid \mathrm{H}_{i}, \mathbf{a}, \sigma^{2}, \alpha=\alpha_{i}\right) \\
& \cong \frac{1}{\pi^{N} \sigma^{2 N}} \exp \left\{-\frac{1}{\sigma^{2}} \sum_{n=M+1}^{N}\left|y_{n}^{i}-\sum_{j=1}^{M} a_{j} y_{n-j}^{i}\right|^{2}\right\} \\
& i=0,1, \\
& L(\mathbf{y})=\frac{f_{y}\left(\mathbf{y} \mid \mathrm{H}_{1}, \mathbf{a}, \sigma^{2}, \alpha\right)}{f_{y}\left(\mathbf{y} \mid \mathrm{H}_{0}, \mathbf{a}, \sigma^{2}, \alpha=0\right)}
\end{aligned}
$$

where:

$$
\begin{aligned}
& y_{n}^{i}= \begin{cases}y_{n} & \text { under } \mathrm{H}_{0}(i=0) \\
y_{n}-\alpha s_{n} & \text { under } \mathrm{H}_{1}(i=1)\end{cases} \\
& \alpha_{i}= \begin{cases}0 & \text { under } \mathrm{H}_{0}(i=0) \\
\alpha & \text { under } \mathrm{H}_{1}(i=1)\end{cases}
\end{aligned}
$$

and $s_{n}$ is the $n$th element of vector $s$. Note that the implementation of the optimum Neyman-Pearson detector requires perfect knowledge of the interference covariance matrix as well as the target amplitude [28]. Here, due to the unknown parameters $\left\{\mathbf{a}, \sigma^{2}, \alpha\right\}$ in the above ratio, realization of the optimum NeymanPearson detector is not possible. However, with a proper estimation of these parameters under any hypothesis, we can obtain a suboptimum detector with the following test statistic:

$$
L_{\text {subopt }}(\mathbf{y})=\frac{f_{y}\left(\mathbf{y} \mid \mathrm{H}_{1}, \hat{\mathbf{a}}_{\mathbf{1}}, \hat{\sigma}_{1}^{2}, \hat{\alpha}\right)}{f_{y}\left(\mathbf{y} \mid \mathrm{H}_{0}, \hat{\mathbf{a}}_{0}, \hat{\sigma}_{0}^{2}, \alpha=0\right)} \gtrless_{\mathrm{H}_{0}}^{\mathrm{H}_{1}} \eta .
$$

The ML estimates of $\alpha$ under $\mathrm{H}_{1}$ and also $\sigma^{2}$ under $\mathrm{H}_{1}$ and $\mathrm{H}_{0}$ were used so that the test statistic (5) would yield the following [12]:

$$
\begin{aligned}
& \frac{\hat{\sigma}_{0}^{2}}{\hat{\sigma}_{1}^{2}} \gtrless_{\mathrm{H}_{0}}^{\mathrm{H}_{1}} \eta, \\
& \hat{\sigma}_{0}^{2}=\frac{1}{N}\left\|\mathbf{u}-\mathbf{Y} \hat{\mathbf{a}}_{0}\right\|^{2}, \\
& \hat{\sigma}_{1}^{2}=\frac{1}{N}\left\|\mathbf{u}^{\prime}-\mathbf{Y}^{\prime} \hat{\mathbf{a}}_{1}\right\|^{2},
\end{aligned}
$$

where:

$$
\mathbf{u}=\left[\begin{array}{lll}
y_{M+1} & \cdots & y_{N}
\end{array}\right]^{T}
$$




$$
\begin{aligned}
& \mathbf{Y}=\left[\begin{array}{ccc}
y_{M} & \cdots & y_{1} \\
\vdots & \ddots & \vdots \\
y_{N-1} & \cdots & y_{N-M}
\end{array}\right] \\
& \mathbf{u}^{\prime}=\mathbf{H u}, \quad \mathbf{Y}^{\prime}=\mathbf{H Y}
\end{aligned}
$$

where $\mathbf{H}$ is the projection matrix of the null space of $\psi$ which is defined as:

$$
\begin{aligned}
& \mathbf{H}=\mathbf{I}-\frac{\boldsymbol{\psi} \boldsymbol{\psi}^{H}}{\boldsymbol{\psi}^{H} \boldsymbol{\psi}}, \\
& \boldsymbol{\psi}=\left[\begin{array}{lllll}
1 & e^{j \theta} & e^{j 2 \theta} & \cdots & e^{j(N-M-1) \theta}
\end{array}\right]^{T} .
\end{aligned}
$$

The superscript $H$ denotes the conjugate transpose operator, and $\mathbf{I}$ is the identity matrix. In addition, the noise variances namely $\hat{\sigma}_{0}^{2}$ and $\hat{\sigma}_{1}^{2}$ under $\mathrm{H}_{0}$ and $\mathrm{H}_{1}$ can be obtained by substituting the ML estimations $\hat{\mathbf{a}}_{0}$ and $\hat{\mathbf{a}}_{1}$ in Eqs. (7) and (8). Then, by substituting $\hat{\sigma}_{0}^{2}$ and $\hat{\sigma}_{1}^{2}$ into Eq. (6), the GLRT detector based on the ML estimation of unknown parameters is derived and called ARGLR [12].

This study used the Kalman-based filter for estimating the coefficients $\hat{\mathbf{a}}_{0}$ and $\hat{\mathbf{a}}_{1}$, employed to finalize test Eq. (6) and proposed a new detector. Of note, in [26], the coefficients of the AR model were estimated using the conventional KF approximately, and the achieved detector was called ARKD. Since the data model is non-linear, the EKF and UKF were used for estimating the AR model in this paper and the results revealed that the obtained detectors exhibited better performance.

\section{Detection based on Kalman estimates of interference parameters}

In this section, the EKF and UKF are employed to estimate the unknown coefficients of the AR model used in Eqs. (7) and (8) and substitute these estimates in Eq. (6) which resulted in two new detectors. Modeling the interference as an AR process provides a group of equations that are appropriate for better realization of the Kalman family filters with high accuracy and ability in estimating the unknown parameters of AR model. The first stage in achieving $\mathrm{KF}$ is to define the state variables and obtain the suitable Gauss-Markov equations. As mentioned earlier, $\mathbf{y}=$ $\left[\begin{array}{llll}y_{1} & y_{2} & \cdots & y_{N}\end{array}\right]^{T}$ denotes the data vector received from the CUT and contains the interference $\mathbf{n}=$ $\left[\begin{array}{llll}n_{1} & n_{2} & \cdots & n_{N}\end{array}\right]^{T}$. Under both hypotheses, we can write $n_{n}=y_{n}-\alpha s_{n}, n=1, \cdots, N$ (note that under $\mathrm{H}_{0}, \alpha=0$ ) and substitute it into Eq. (2) which results in the following recursive relation:

$$
\begin{aligned}
y_{n}= & a_{1}\left(y_{n-1}-\alpha s_{n-1}\right)+a_{2}\left(y_{n-2}-\alpha s_{n-2}\right) \\
& +a_{M}\left(y_{n-M}-\alpha s_{n-M}\right)+\alpha s_{n}+w_{n} .
\end{aligned}
$$

In this equation, $w_{n}$ is the complex white Gaussian noise of the AR model with variance $\sigma^{2}$, and $\alpha$ and $s_{n}$ are the complex amplitudes and the $n$th element of the target vector, respectively. For indices $n \leq 0, y_{n}$ and $s_{n}$ are considered equal to zero. Here, $\alpha$ and $a_{1}$ to $a_{M}$ are unknown parameters; hence, the state vector at time $n$ is considered as $\mathbf{x}_{n}=\left[\begin{array}{lllll}a_{1 n} & a_{2 n} & \cdots & a_{M n} & \alpha_{n}\end{array}\right]^{T}=$ $\left[\begin{array}{ll}\mathbf{a}_{n}^{T} & \alpha_{n}\end{array}\right]^{T}$. As observed in Eq. (14), $y_{n}$ cannot be displayed as a linear combination of state variables which is the main idea behind using the EKF and UKF. In fact, the measurement equation has a nonlinear form as follows:

$$
y_{n}=h_{n}\left(\mathbf{x}_{n}\right)+w_{n} .
$$

However, in order to obtain the other equation of Gauss-Markov model, we assume that during a CPI, the AR model coefficients belonging to the interference of the CUT are almost time invariant. Moreover, the slow target fluctuation was taken into consideration to fix the target amplitude. Therefore, the state vector is assumed to be fixed within the CPI, and the system dynamic equation can be written as follows:

$$
\mathbf{x}_{n+1}=\mathbf{x}_{n}+\mathbf{v}_{n}
$$

where $\mathbf{v}_{n}$ is a complex white Gaussian noise with covariance matrix $\mathbf{Q}_{n}$, as shown in the following:

$$
\mathbf{Q}_{n}=\left[\begin{array}{cc}
q_{n}^{2} \mathbf{I}_{M} & 0 \\
0 & q_{n}^{\prime 2}
\end{array}\right]
$$

where $q_{n}^{2}$ and $q_{n}^{\prime 2}$ are the variances of the deviations of the AR model coefficients and target amplitude, respectively. It is clear that consideration of $\mathbf{v}_{n}$ makes small changes in the AR model coefficients and target signal amplitude during the implementation of the KF steps. Here, the Gauss-Markov equations can be expressed as follows:

$$
\left\{\begin{array}{l}
\mathbf{x}_{n+1}=\mathbf{x}_{n}+\mathbf{v}_{n} \\
y_{n}=h_{n}\left(\mathbf{x}_{n}\right)+w_{n}
\end{array} \quad n=1, \cdots, N .\right.
$$

The nonlinear form of Gauss-Markov equations was taken into account to estimate the state vector $\mathbf{x}_{n}$ based on the EKF and UKF algorithms using only the primary data.

\subsection{Detector based on EKF}

Suppose that $\hat{\mathbf{x}}_{n \mid n-1}$ is the prediction of the state vector $\mathbf{x}_{n}$ based on the received data until time $n-1$, namely $\left[\begin{array}{llll}y_{1} & y_{2} & \cdots & y_{n-1}\end{array}\right]^{T}$. In the EKF, non-linear function $h_{n}\left(\mathbf{x}_{n}\right)$ in Eq. (18) is changed into a linear equation in terms of the state variables using Taylor expansion around the point $\mathbf{x}_{n}=\hat{\mathbf{x}}_{n \mid n-1}$, as shown in the following:

$$
h_{n}\left(\mathbf{x}_{n}\right)=h_{n}\left(\hat{\mathbf{x}}_{n \mid n-1}\right)+\left.\frac{\partial h_{n}}{\partial \mathbf{x}_{n}}\right|_{\mathbf{x}_{n}=\hat{\mathbf{x}}_{n \mid n-1}}\left(\mathbf{x}_{n}-\hat{\mathbf{x}}_{n \mid n-1}\right) .
$$


In this case, since the variation $\left(\mathbf{x}_{n}-\hat{\mathbf{x}}_{n \mid n-1}\right)$ becomes small, the second and higher order terms in the expansion are supposed to be negligible. By defining:

$$
\mathbf{H}_{n}=\left.\frac{\partial h_{n}}{\partial \mathbf{x}_{n}}\right|_{\mathbf{x}_{n}=\hat{\mathbf{x}}_{n \mid n-1}}
$$

and through the definition $h_{n}\left(\mathbf{x}_{n}\right)$ in Eq. (15), we have:

$$
\mathbf{H}_{n}=\left[\begin{array}{c}
y_{n-1}-\hat{\alpha}_{n \mid n-1} s_{n-1} \\
y_{n-2}-\hat{\alpha}_{n \mid n-1} s_{n-2} \\
\vdots \\
y_{n-M}-\hat{\alpha}_{n \mid n-1} s_{n-M} \\
s_{n}-\hat{\mathbf{a}}_{1 n \mid n-1} s_{n-1}-\hat{\mathbf{a}}_{2 n \mid n-1} s_{n-2}-\ldots \\
-\hat{\mathbf{a}}_{M n \mid n-1} s_{n-M}
\end{array}\right]^{T}
$$

Thus, the equations of the EKF in the measurementupdate algorithm denoted by $\hat{\mathbf{x}}_{n \mid n}$ are given below:

$$
\begin{aligned}
& \hat{\mathbf{x}}_{n \mid n}=\hat{\mathbf{x}}_{n \mid n-1}+\mathbf{L}_{n}\left(y_{n}-h_{n}\left(\hat{\mathbf{x}}_{n \mid n-1}\right)\right), \\
& \mathbf{L}_{n}=\mathbf{P}_{n \mid n-1} \mathbf{H}_{n}\left(\mathbf{H}_{n}^{H} \mathbf{P}_{n \mid n-1} \mathbf{H}_{n}+\sigma_{n}^{2}\right)^{-1}, \\
& \mathbf{P}_{n \mid n}=\left(\mathbf{I}-\mathbf{L}_{n} \mathbf{H}_{n}^{H}\right) \mathbf{P}_{n \mid n-1},
\end{aligned}
$$

where $\sigma_{n}^{2}$ is the variance of the white noise $w_{n}$ in the AR models (2) and (18) that can be time-varying in the proposed method.

According to the linear form of the system equation in Eq. (18), the equations of the EKF in the timeupdate algorithm or prediction are as follows:

$$
\begin{aligned}
& \hat{\mathbf{x}}_{n \mid n-1}=\hat{\mathbf{x}}_{n-1 \mid n-1}, \\
& \mathbf{P}_{n \mid n-1}=\mathbf{P}_{n-1 \mid n-1}+\mathbf{Q}_{n} .
\end{aligned}
$$

In the above equations, $\mathbf{P}$ denotes the covariance matrix of that state estimate in each stage. Since the variance $\sigma_{n}^{2}$ and covariance matrix $\mathbf{Q}_{n}$ are not available in a practical sense, during the implementation of the $\mathrm{KF}$ algorithm, we can adaptively estimate these values with moment estimation. Based on Eq. (15), $\sigma_{n}^{2}$ can be written as follows:

$$
\sigma_{n}^{2}=E\left\{\left(y_{n}-h_{n}\left(\mathbf{x}_{n}\right)\right)^{H}\left(y_{n}-h_{n}\left(\mathbf{x}_{n}\right)\right)\right\},
$$

where $E$ denotes the expectation value operator. The moment estimation of $\sigma_{n}^{2}$ is obtained as follows [9]:

$$
\begin{aligned}
\hat{\sigma}_{n}^{2}= & \frac{1}{K} \sum_{k=n-K+1}^{n} E\left\{\left(y_{k}-h_{k}\left(\hat{\mathbf{x}}_{k \mid k-1}\right)\right)^{H}\right. \\
& \left.\left(y_{k}-h_{k}\left(\hat{\mathbf{x}}_{k \mid k-1}\right)\right)\right\} .
\end{aligned}
$$

Based on the simulation investigation, we suggest $K=$ 3 which should be temporarily assumed to be $K=n$ for $n<2$ to prevent $k$ from being negative. Similarly, through Eq. (16), $\mathbf{Q}_{n}$ can be expressed as follows:

$$
\mathbf{Q}_{n}=E\left\{\left(\mathbf{x}_{n+1}-\mathbf{x}_{n}\right)\left(\mathbf{x}_{n+1}-\mathbf{x}_{n}\right)^{H}\right\} \text {. }
$$

Thus, the moment estimation of $Q_{n}$ can be obtained as follows:

$$
\begin{gathered}
\hat{\mathbf{Q}}_{n}=\frac{1}{K} \sum_{k=n-K+1}^{n} E\left\{\left(\hat{\mathbf{x}}_{k+1 \mid k+1}-\hat{\mathbf{x}}_{k \mid k}\right)\right. \\
\left.\left(\hat{\mathbf{x}}_{k+1 \mid k+1}-\hat{\mathbf{x}}_{k \mid k}\right)^{H}\right\},
\end{gathered}
$$

where $K$ is supposed to be acting as before.

After running the EKF, the AR model coefficients (of the interference) are estimated, and by substituting them in Eqs. (7) and (8), the noise variance of the AR model is estimated under both hypotheses as follows:

$$
\begin{aligned}
& \hat{\sigma}_{0}^{2}=\frac{1}{N}\left\|\mathbf{u}-\mathbf{Y} \hat{\mathbf{a}}_{N \mid N}\right\|^{2}, \\
& \hat{\sigma}_{1}^{2}=\frac{1}{N}\left\|\mathbf{u}^{\prime}-\mathbf{Y}^{\prime} \hat{\mathbf{a}}_{N \mid N}\right\|^{2} .
\end{aligned}
$$

Finally, by substituting $\hat{\sigma}_{0}^{2}$ and $\hat{\sigma}_{1}^{2}$ in Eq. (6), the first proposed detector can be obtained called AREKF.

\subsection{Detector based on UKF}

Unlike the EKF, UKF has no need for equation linearization and the required means for implementing the KF algorithm is obtained by simulation. Assuming that we have $l$ state variables, to run each step of the filter, we use $M=2 l+1$ weighted samples (called sigma points) whose weights are determined as follows:

$$
w_{0}=\frac{\lambda}{l+\lambda}, \quad w_{i}=\frac{1}{2(l+\lambda)}, \quad i=1,2, \cdots, 2 l .
$$

Moreover, in each step of the algorithm, the sigma points are defined as follows:

$$
\begin{aligned}
& \chi_{0, n-1 \mid n-1}=\hat{\mathbf{x}}_{n-1 \mid n-1}, \\
& \chi_{i, n-1 \mid n-1}=\hat{\mathbf{x}}_{n-1 \mid n-1}+\left[\sqrt{(l+\lambda) \mathbf{P}_{n-1 \mid n-1}}\right]_{i}, \\
& i=1,2, \cdots, l, \\
& \chi_{i, n-1 \mid n-1}=\hat{\mathbf{x}}_{n-1 \mid n-1}-\left[\sqrt{(l+\lambda) \mathbf{P}_{n-1 \mid n-1}}\right]_{i}, \\
& i=l+1, \cdots, 2 l,
\end{aligned}
$$

where $[\mathbf{A}]_{i}$ denotes the $i$ th column of matrix $\mathbf{A}$ and the square root of the covariance matrix $\mathbf{P}_{n-1 \mid n-1}$ is obtained by Cholesky factorization. In addition, $\lambda$ is a scaling parameter and an appropriate choice of it can 
reduce the estimation error. In [29], this parameter is proposed as follows:

$$
\lambda=3 \rho^{2}-l,
$$

where $\rho$ is a constant that determines the spread of the samples around the estimates of state vector at each step, and it is usually set to a small positive value (e.g., $\left.10^{-4} \leq \rho \leq 1\right)$.

According to the system dynamics presented in Eq. (18), the weighted samples do not change and the equations of the UKF in the time-update algorithm are as follows:

$$
\begin{aligned}
\chi_{i, n \mid n-1}= & \chi_{i, n-1 \mid n-1}, \quad i=0,1, \cdots, 2 l, \\
\hat{\mathbf{x}}_{n \mid n-1}= & \sum_{i=0}^{2 l} w_{i} \chi_{i, n \mid n-1}, \\
\mathbf{P}_{n \mid n-1}= & \sum_{i=0}^{2 l} w_{i}\left(\chi_{i, n \mid n-1}-\hat{\mathbf{x}}_{n \mid n-1}\right) \\
& \left(\chi_{i, n \mid n-1}-\hat{\mathbf{x}}_{n \mid n-1}\right)^{H}+\mathbf{Q}_{n} .
\end{aligned}
$$

Similarly, the equations of the UKF in the measurement-update algorithm are given as follows:

$$
\begin{aligned}
& \hat{\mathbf{x}}_{n \mid n}=\hat{\mathbf{x}}_{n \mid n-1}+K_{n}\left(y_{n}-\hat{y}_{n \mid n-1}\right) \\
& \hat{y}_{n \mid n-1}=\sum_{i=0}^{2 l} w_{i} \zeta_{i, n \mid n-1}, \\
& \zeta_{i, n \mid n-1}=h_{n}\left(\chi_{i, n \mid n-1}\right) \\
& \mathbf{K}_{n}=\mathcal{P}_{x \zeta, n \mid n-1} \mathcal{P}_{\zeta \zeta, n \mid n-1}^{-1}, \\
& \mathcal{P}_{x \zeta, n \mid n-1}=\sum_{i=0}^{2 l} w_{i}\left(\chi_{i, n \mid n-1}-\hat{\mathbf{x}}_{n \mid n-1}\right) \\
& \left(\zeta_{i, n \mid n-1}-\hat{\mathbf{y}}_{n \mid n-1}\right)^{H}, \\
& \mathcal{P}_{\zeta \zeta, n \mid n-1}=\sum_{i=0}^{2 l} w_{i}\left(\zeta_{i, n \mid n-1}-\hat{\mathbf{y}}_{n \mid n-1}\right) \\
& \left(\zeta_{i, n \mid n-1}-\hat{\mathbf{y}}_{n \mid n-1}\right)^{H}, \\
& \mathbf{P}_{n \mid n}=\mathbf{P}_{n \mid n-1}-\mathcal{P}_{x \zeta, n \mid n-1} \mathcal{P}_{\zeta \zeta, n \mid n-1}^{-1} \mathcal{P}_{x \zeta, n \mid n-1}^{H}
\end{aligned}
$$

Here, during the implementation of the UKF algorithm, like the EKF, we can adaptively estimate the variance $\sigma_{n}^{2}$ and covariance matrix $\mathbf{Q}_{n}$ using moment estimation.

By the same token, after running the UKF, the AR model coefficients (of the interference) were estimated and by substituting them in Eqs. (24) and (25), the noise variance of the AR model was estimated under both hypotheses. Finally, upon substituting the obtained $\hat{\sigma}_{0}^{2}$ and $\hat{\sigma}_{1}^{2}$ into Eq. (6) using the UKF, the second proposed detector is achieved, which is called ARUKF.

The state space model in Eq. (18) is a nonlinear model with time-variant parameters, and this complexity shows that the convergence and stability verification of the algorithm is not analytically easy to do. Therefore, the stability of an algorithm was evaluated using simulation and statistical surveys under different conditions. The obtained results point to the high stability of the proposed methods.

In the next section, the simulation results are employed to evaluate the performance of the proposed detectors and compare it with that of ARGLR and ARKD detectors. According to the findings, the ARUKF detector outperforms its counterparts, especially on the low sample support.

\section{Performance evaluation}

In this section, by resorting to Monte Carlo simulation, the performance of the proposed detectors is evaluated and compared with that of ARKD and ARGLR. In modeling the ground clutter through an AR process, the model order is usually assumed to be less than four. In our simulations, the interference is regarded as an AR Gaussian process of order 2 with coefficients vector $\mathbf{a}=\left[\begin{array}{ll}-0.25+0.25 i & 0.3\end{array}\right]^{T}$ in [12]. An arbitrary variance for the interference was taken into account to determine the AR model of the interference and its experimental covariance matrix $\mathbf{R}_{N}$, which is obtained by averaging a sufficient number of the simulated interference vectors. The signal-to-interference ratio is defined as follows [12]:

$$
S N R=|\alpha|^{2} \mathbf{s}^{H} \mathbf{R}_{N}^{-1} \mathbf{s} .
$$

The target vector is assumed to be known, the target amplitude is achieved in simulations for each SNR. Also, the target phase is determined as a random value with uniform distribution over the range $[-\pi,+\pi)$. For evaluating and comparing the detection performance, the Receiver Operation Characteristic (ROC) curves of the detectors are used. In order to ensure a precise evaluation, the curves of detection probability versus SNR are plotted as well.

Before comparing the detectors with each other, we first study the performance of each of the two proposed detectors by changing the number of primary data $N$. For this purpose, the curves of detection probability versus the probability of false alarm and those versus the signal to noise ratio are plotted at different values of $N$. 
Figure 1 shows the probability of detection $\left(P_{d}\right)$ versus the probability of false alarm $\left(P_{f a}\right)$ of AREKF detector at different values of $N, \theta=1 \mathrm{rad}$ and $S N R=$ $10 \mathrm{~dB}$. As it can be seen, with the increase in the number of primary data, due to a more accurate estimation of the interference parameters, the performance of detector is enhanced. Figure 2 shows the AREKF detection probability versus SNR at different values of $N, \theta=1 \mathrm{rad}$ and $P_{f a}=10^{-2}$. This figure shows that at first, by increasing the SNR, the probability of detection is enhanced; however, if this ratio exceeds a certain limit owing to the decline in the relative power of the AR interference, the estimation error associated with the AR model parameters increases and the probability of detection declines. However, with the increasing number of primary data, this event occurs later or, in other words, occurs at larger values of SNR.

Next, in Figures 3 and 4, the performance of ARUKF detector is considered by changing the number of primary data. In Figure 3 , the curves of $P_{d}$ versus $P_{f a}$ at different values of $N, \theta=1 \mathrm{rad}$ and

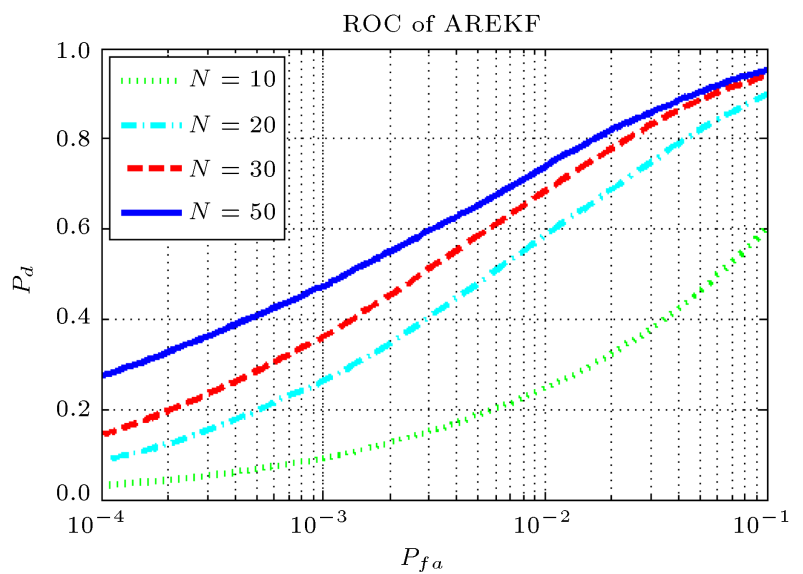

Figure 1. $P_{d}$ against $P_{f a}$ for AREKF with $S N R=10 \mathrm{~dB}$, $\theta=1 \mathrm{rad}$ and four values of $N$.

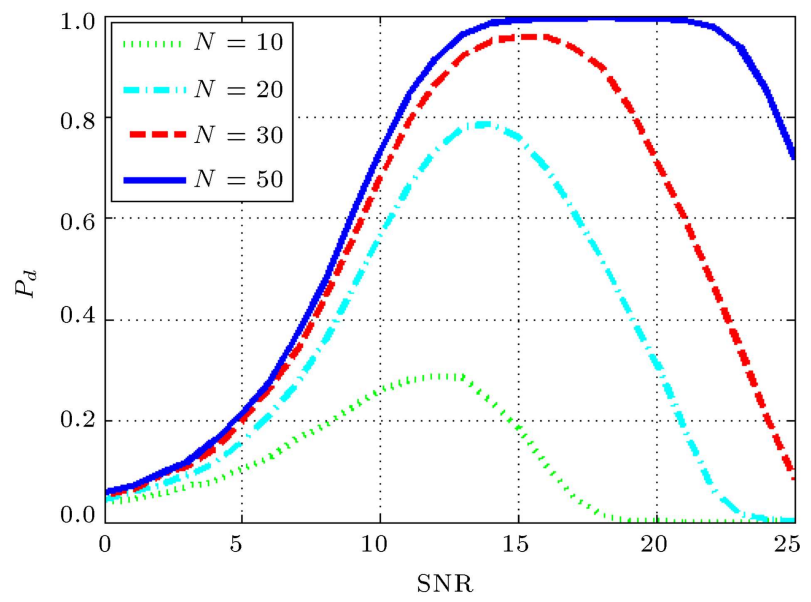

Figure 2. $P_{d}$ against Signal-to-Noise Ratio (SNR) for AREKF with $P_{f a}=10^{-2}, \theta=1 \mathrm{rad}$ and four values of $N$.
$S N R=10 \mathrm{~dB}$ are comparatively plotted. As expected, owing to the dependence of the estimation accuracy on the number of primary data, a greater increase in the number of primary data, provides a greater improvement in the detector performance. Figure 4 plots the curve of $P_{d}$ versus SNR at different values of $N, \theta=1 \mathrm{rad}$ and $P_{f a}=10^{-2}$. Fortunately, even for the small number of primary data sets, by increasing SNR up to $25 \mathrm{~dB}$, the detection probability will not decline. Indeed, even for the small number of primary data sets, the estimation error of the AR model parameters in the UKF does not increase with a rise in the SNR value. This is a significant advantage of ARUKF detector compared with the previous detectors based on KF such as AREKF and ARKD.

In the following, by using the ROC curves as well as the curves of detection probability versus $S N R$, the performances of the proposed AREKF and ARUKF detectors is compared with those of ARGLR and ARKD detectors. Figures 5, 6, and 7 show the ROC curves of these detectors for different number of primary datasets

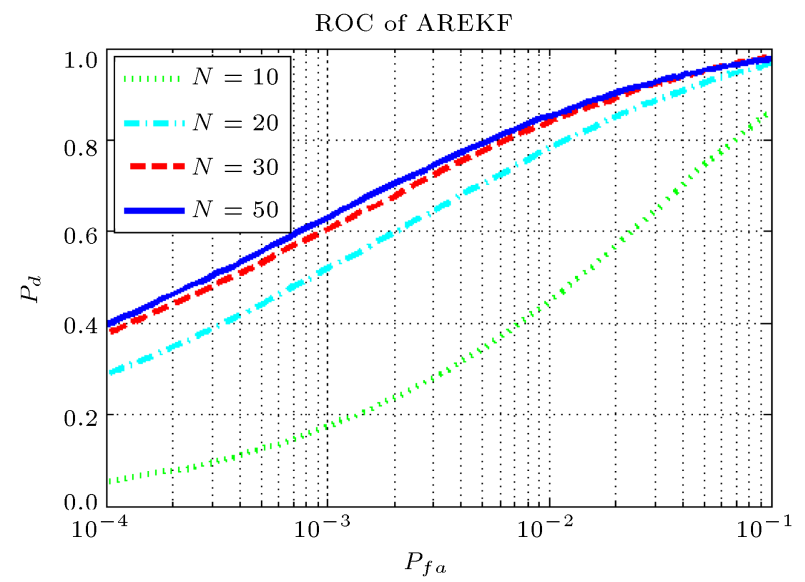

Figure 3. $P_{d}$ against $P_{f a}$ for ARUKF with $S N R=10 \mathrm{~dB}$, $\theta=1 \mathrm{rad}$ and four values of $N$.

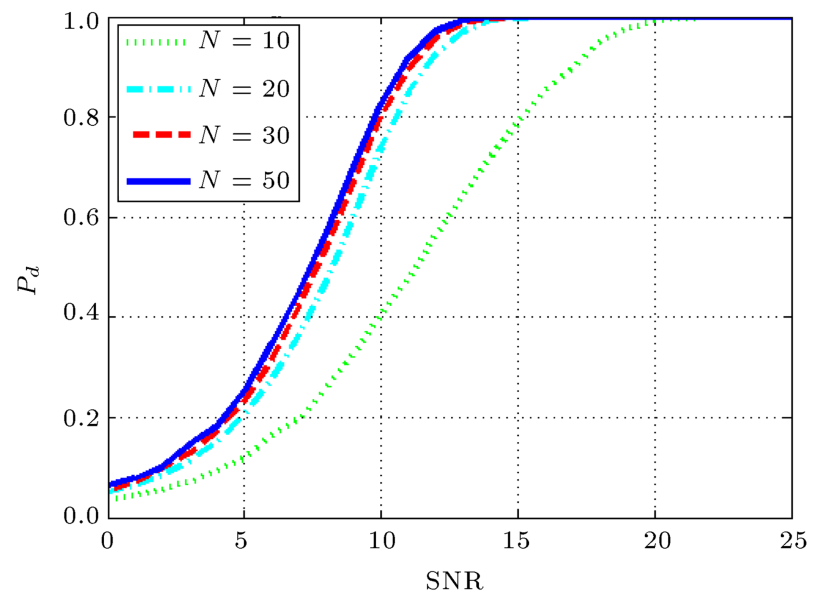

Figure 4. $P_{d}$ against Signal-to-Noise Ratio (SNR) for ARUKF with $P_{f a}=10^{-2}, \theta=1 \mathrm{rad}$ and four values of $N$. 


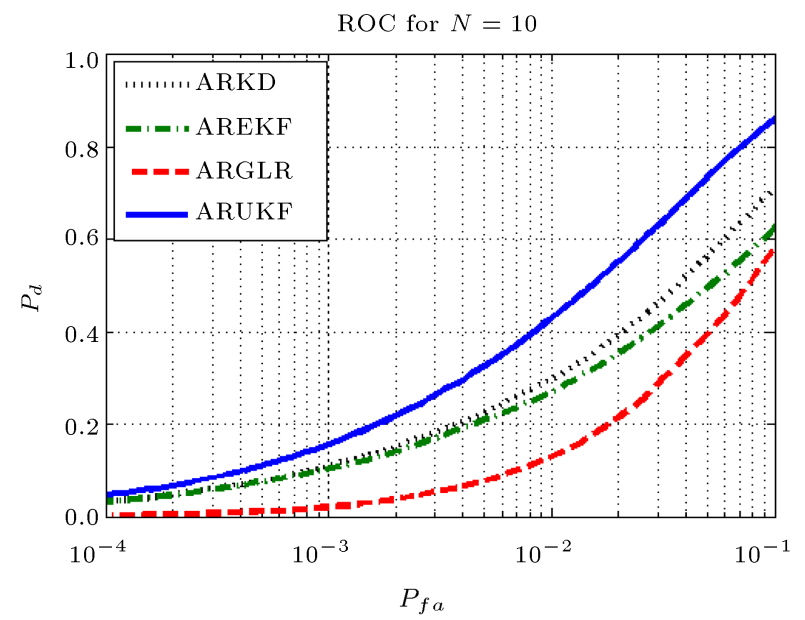

Figure 5. $P_{d}$ against $P_{f a}$ for AREKF and ARUKF compared with ARGLR and ARKD for $S N R=10 \mathrm{~dB}$, $\theta=1 \mathrm{rad}$, and $N=10$.

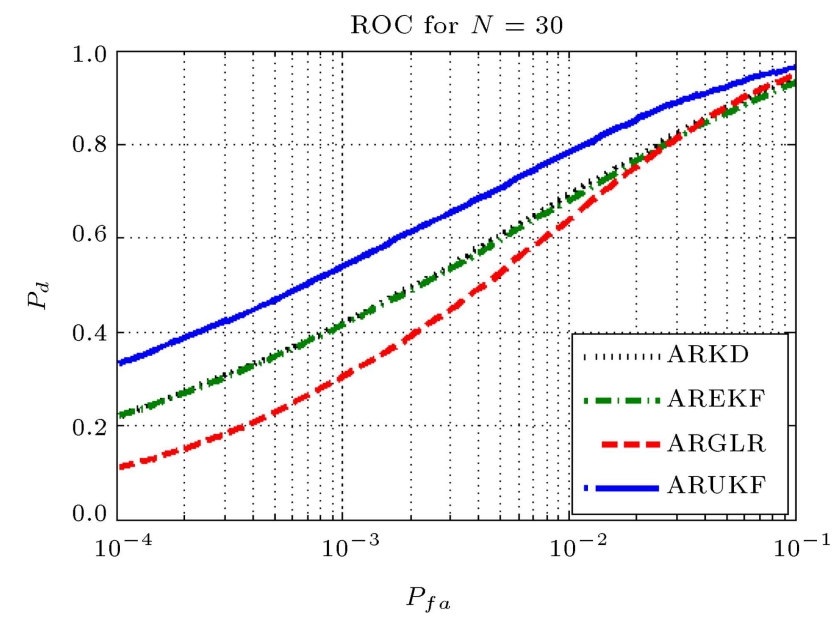

Figure 6. $P_{d}$ against $P_{f a}$ for AREKF and ARUKF compared with ARGLR and ARKD for $S N R=10 \mathrm{~dB}$, $\theta=1 \mathrm{rad}$, and $N=30$.

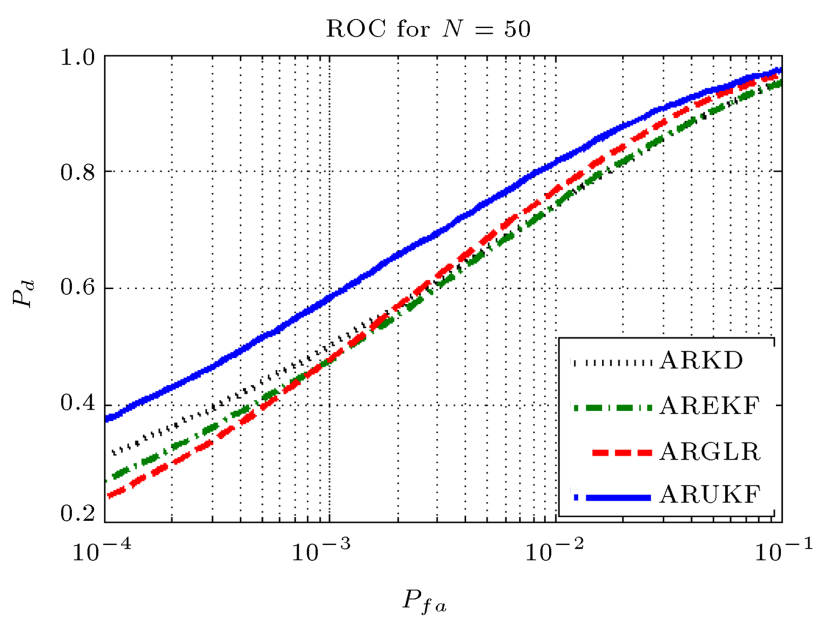

Figure 7. $P_{d}$ against $P_{f a}$ for AREKF and ARUKF compared with ARGLR and ARKD for $S N R=10 \mathrm{~dB}$, $\theta=1 \mathrm{rad}$, and $N=50$.
$N=10, N=30$, and $N=50$, respectively, at $\theta=1 \mathrm{rad}$ and $S N R=10 \mathrm{~dB}$. It is observed that in the case of small number of primary data set, the KF-based detectors outperform the ARGLR detector and especially the ARUKF always has considerably better performance than that of other detectors. We see that with increase in the number of primary data, the ARGLR performance will gradually become better than the performance of ARKD and AREKF, because the AR model parameters in ARGLR are estimated entirely based on the ML method and, hence, with increase in the number of data, these estimates will tend to the efficient estimates [30].

In Figures 8, 9, and 10, the curves of detection probability versus SNR of the mentioned detectors for different numbers of primary data including $N=10$, $N=30$, and $N=50, \theta=1 \mathrm{rad}$, and $P_{f a}=10^{-2}$ are plotted. Here again, we see that the performance

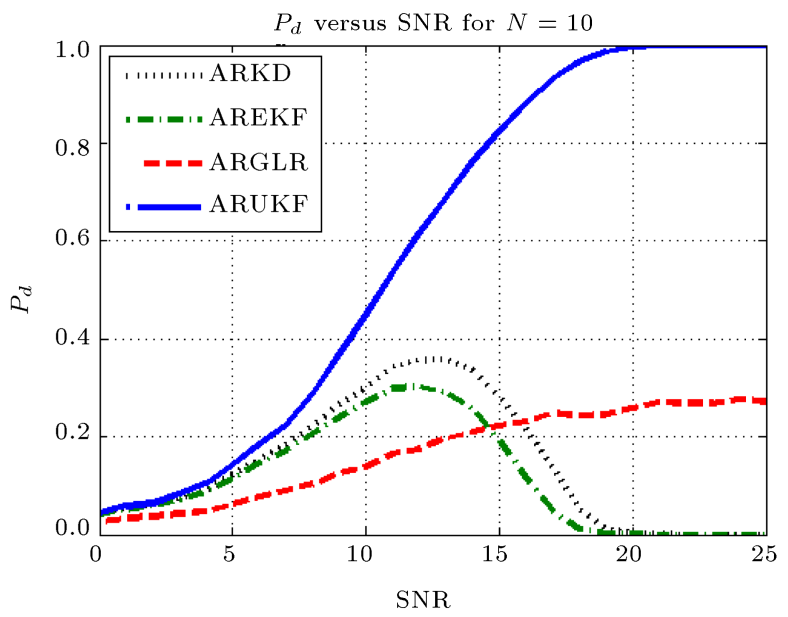

Figure 8. $P_{d}$ against Signal-to-Noise Ratio (SNR) for AREKF and ARUKF compared with ARGLR and ARKD for $P_{f a}=10^{-2}, \theta=1 \mathrm{rad}$, and $N=10$.

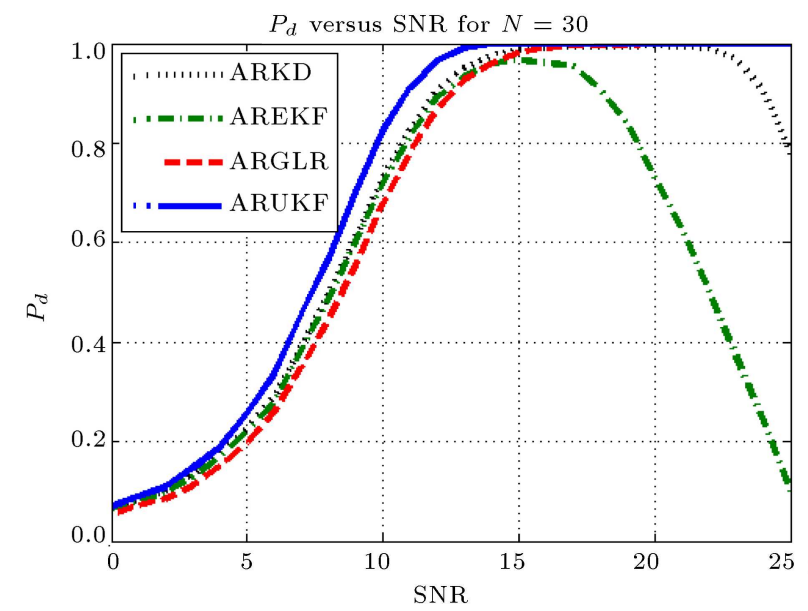

Figure 9. $P_{d}$ against Signal-to-Noise Ratio (SNR) for AREKF and ARUKF compared with ARGLR and ARKD for $P_{f a}=10^{-2}, \theta=1 \mathrm{rad}$, and $N=30$. 


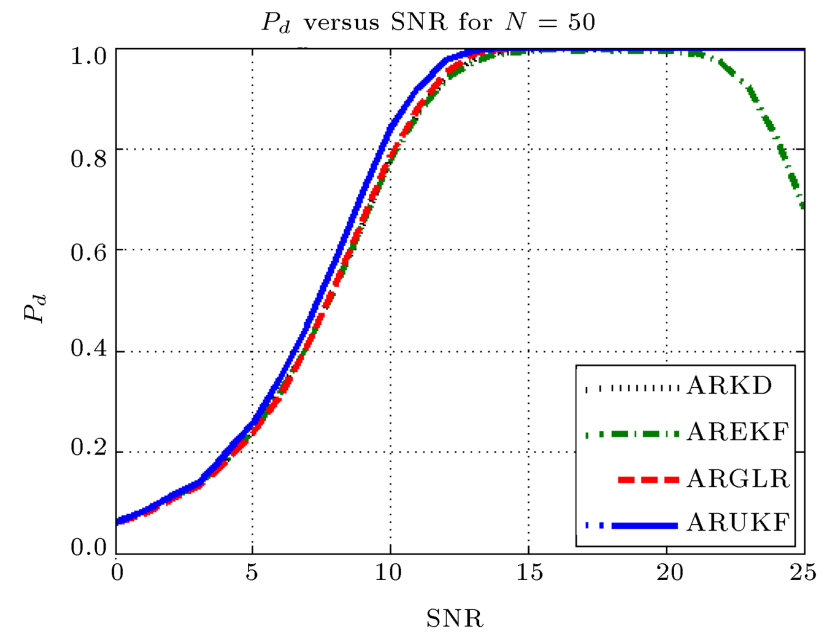

Figure 10. $P_{d}$ against Signal-to-Noise Ratio (SNR) for AREKF and ARUKF compare with ARGLR and ARKD for $P_{f a}=10^{-2}, \theta=1 \mathrm{rad}$ and $N=50$.

of ARUKF detector significantly is better than that of others. However, with increase in the number of primary data sets, the parameter estimation error for the AR model is reduced which is the reason why the performances of other detectors, especially ARGLR, approach those of the ARUKF. Interestingly, as considered and discussed in Figures 2 and 4, the detection probability of ARUKF will not fall by increasing SNR even for small number of primary data sets. This is another important advantage of the ARUKF detector compared with the two other KF-based detectors.

We also investigate the convergence of the KFbased detectors. For this purpose, the Mean Squared Error (MSE) of the estimates of AR model coefficients is considered. Figure 11, obtained by simulation results in different conditions, shows the MSE of estimates versus the number of primary data sets for two situations: the first is noise only and the second considers the signal with $S N R=25 \mathrm{~dB}$. As is seen, following an

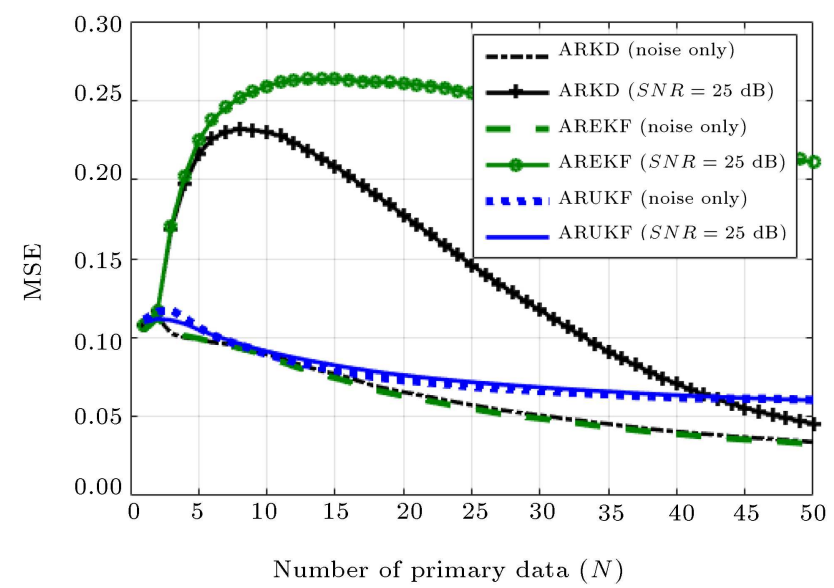

Figure 11. Mean Squared Error (MSE) versus $N$ for noise only and signal with $S N R=25 \mathrm{~dB}$. initial transition (almost 3 steps for the absent signal), the MSE is monotonically reduced by increasing the number of data sets in all detectors. This shows the convergence of all of the algorithms. In the first situation, all methods have a relatively small error although the MSE of the ARUKF is a little more than those of the other two detectors. In the second situation with $S N R=25 \mathrm{~dB}$, the MSEs and transient times of the ARKD and AREKF have significantly increased in comparison with the first situation, while the MSE of the ARUKF has a slight increase and its transient time has even been reduced to 2 . The increase in the initial error results from the KF transient state and, after passing through this transient state, the error decreases with increase in the number of data sets and the estimate of each method converges to a final value. The interesting advantage of the ARUKF is that the transient state error of the filter is relatively low and this results in a better performance of this method. In the following convergence study, an analysis with one million iterations in different conditions shows that the error of each method in each iteration is always less than one and no instability is seen during the estimation.

Finally, the effect of Doppler shift frequency error of the target on the performance of detectors is investigated. It is common in radar signal detection to divide the target velocity (or Doppler frequency) domain into small cells and test each cell for the presence of target [27]. For simulation, the nominal $\theta$ is assumed to be $\theta_{\text {nom }}=1 \mathrm{rad}$ in the detectors while the data vector $y$ is simulated with the actual $\theta$ with a $5 \%$ difference compared to the nominal $\theta_{\text {nom }}=1 \mathrm{rad}$. Figure 12 shows the ROC curve of the mentioned detectors at $N=30, \theta_{\text {nom }}=1 \mathrm{rad}$, and $S N R=10 \mathrm{~dB}$. A comparison of the curves in Figures 6 and 12 shows that although the presence of an error in Doppler shift

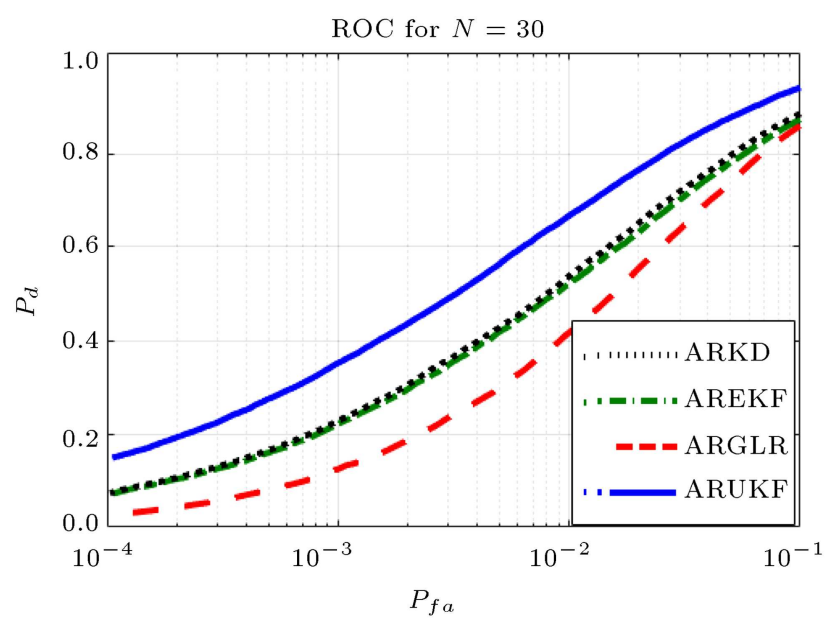

Figure 12. Receiver Operation Characteristic (ROC) curve of the detectors for $S N R=10 \mathrm{~dB}, \theta_{\text {nom }}=1 \mathrm{rad}$ and $N=30$. 
causes loss in the performance of the detectors, the performance of the ARUKF is also better than those of other detectors.

\section{Conclusion}

This study applied the adaptive detection of a radar target signal embedded in Gaussian interference based on only the primary data where the interference was modeled as an AR process. Herein, ARGLR detector relying on Maximum likelihood (ML) estimation of the AR model parameters and also ARKD detector relying on Kalman estimation of them were developed. In this regard, two new detectors were proposed: AREKF based on Extended KF (EKF) and ARUKF based on Unscented KF (UKF). We demonstrated that the ARUKF detector had significantly better performance than that of other detectors. However, with increase in the number of primary data, parameter estimation error in the AR model is reduced and, hence, the performance of the other detectors, especially ARGLR, approaches that of ARUKF. In addition, a considerable advantage of the ARUKF detector compared to both other KF-based detectors is that the detection probability of ARUKF is not corrupted by increasing Signal-to-Noise Ratio (SNR) even for small numbers of primary data sets; therefore, this detector outperforms its counterpart, especially on the low sample support. At the end, in order to generalize the proposed method, it is suggested that this method be developed using secondary data.

\section{References}

1. Kelly, E.J. "An adaptive detection algorithm", IEEE Trans. Aerosp. Electron. Syst., 22(2), pp. 115-127 (1986).

2. Robey, F.C., Fuhrman, D.L., Kelly, E.J., et al. "A CFAR adaptive matched filter detector", IEEE Trans. Aerosp. Electron. Syst., 29(1), pp. 208-216 (1992).

3. Rafie, A.H. and Taban, M.R. "Two dimensional optimal linear detector for slowly fluctuating radar signals in compound Gaussian clutter", Scientia Iranica, 21(6), pp. 2213-2223 (2014).

4. Conte, E., De Maio, A., and Ricci, G. "GLRTbased adaptive detection algorithms for range-spread targets", IEEE Trans. Signal Process., 49(7), pp. 1336-1348 (2001).

5. Himed, B. and Melvin, W.L. "Analyzing space-time adaptive processors using measured data", Thirty-first Asilomar Conf. on Signals, Systems \& Computers, Pacific Grove, CA, USA, pp. 930-935 (1997).

6. Bandiera, F., Besson, O., Coluccia, A., et al. "ABORTlike detectors: A Bayesian approach", IEEE Trans. Signal Process., 63(19), pp. 5274-5284 (2015).
7. Aubry, A., De Maio, A., Foglia, G., et al. "Diffuse multipath exploitation for adaptive radar detection", IEEE Trans. Signal Process., 63(5), pp. 1268-1281 (2015).

8. Said, S., Hajri, H., Bombrun, L., et al. "Gaussian distributions on riemannian symmetric spaces: statistical kerning with structured covariance matrices", IEEE Trans. Information Theory, 64(2), pp. 752-772 (2018).

9. Kay, S.M. "Asymptotically optimal detection in unknown colored noise via autoregressive modeling", IEEE Trans. on Acoustics, Speech, and Signal Processing, 31(4), pp. 927-940 (1983).

10. Qureshi, T.R., Rangaswamy, M., and Bell, K.L. "Parametric adaptive matched filter for multistatic MIMO radar", IEEE Trans. Aerosp. Electron. Syst., 54(5), pp. 2202-2219 (2018).

11. Haykin, S. and Steinhardt, A., Adaptive Radar Detection and Estimation, Wiley, New York (1992).

12. Sheikhi, A., Nayebi, M.M., and Aref, M.R. "Adaptive detection algorithm for radar signals in autoregressive interference", IEE Proc., Radar Sonar Navig., 145(5), pp. 309-314 (1998).

13. Sheikhi, A., Nayebi, M.M., and Aref, M.R. "A powerful practical coherent adaptive radar detector", 2001 CIE Conf. on Radar, Beijing, China, pp. 405-409 (2001).

14. Moniri, A.R., Nayebi, M.M., and Sheikhi, A. "A multichannel auto-regressive GLR detector for airborne phased array radar applications", 2003 IEEE Conf. on Radar, Adelaide, SA, Australia, pp. 206-211 (2003).

15. Sheikhi, A., Zamani, A., and Norouzi, Y. "Modelbased adaptive target detection in clutter using MIMO radar", 2006 CIE Conf. on Radar, Shanghai, China, pp. 1-4 (2006).

16. Alfano, G., De Maio, A., and Farina, A. "Model-based adaptive detection of range-spread targets", IEE Proc. Radar Sonar Navig., 151(1), pp. 2-10 (2004).

17. Sheikhi, A., Zamani, A., Hatam, M., et al. "Model based adaptive detection algorithm with low secondary data support", 10th Int. Conf. on Information Science, Signal Processing and their Applications, pp. 177-180 (2010).

18. Wang, P., Li, H., and Himed, B. "A parametric moving target detector for distributed MIMO radar in non-homogeneous environment", IEEE Trans. Signal Process., 61(9), pp. 2282-2294 (2013).

19. Li, H., Wang, Z., Liu, J., et al. "Moving target detection in distributed MIMO radar on moving platforms", IEEE J. Select. Topics Signal Process., 9(8), pp. 15241535 (2015).

20. Fan, Y.F., Luo, F., Li, M., et al. "Fractal properties of autoregressive spectrum and its application on weak target detection in sea clutter background", IET Radar Sonar Navigat., 9(8), Kuala Lumpur, Malaysia, pp. 1070-1077 (2015).

21. Sohn, K.J., Li, H., and Himed, B. "Parametric GLRT for multichannel adaptive signal detection", IEEE Trans. Signal Process., 55(11), pp. 5351-5360 (2007). 
22. Wang, P., Li, H., and Himed, B. "A new parametric GLRT for multichannel adaptive signal detection", IEEE Trans. Signal Process., 58(1), pp. 317-325 (2010).

23. Crouse, D.F. "Basic tracking using nonlinear continuous-time dynamic models", IEEE M. Aerosp. Electron. Syst., 30(2), pp. 4-41 (2015).

24. Jahanbakhshi, S., Pishvaie, M.R., and Boozarjomehry, R.B. "Characterization of three-phase flow in porous media using the ensemble Kalman filter", Scientia Iranica, 24(3), pp. 1281-1301 (2017).

25. Kiani, M. and Pourtakdoust, S.H. "Spacecraft attitude and system identification via marginal modified unscented Kalman filter utilizing the sun and calibrated three-axis-magnetometer sensors", Scientia Iranica, 21(4), pp. 1451-1460 (2014).

26. Taban, M.R. and Sheikh Mozaffari, A. "Adaptive radar signal detection in Gaussian clutter with autoregressive model using Kalman filter", Journal of Radar, 2(3), pp. 1-12 (2014).

27. Skolnik, M.I., Introduction to Radar Systems, 3rd Ed., McGraw-Hill, New York (2001).

28. Aubry, A., De Maio, A., Pallotta, L., et al. "Radar detection of distributed targets in homogeneous interference whose inverse covariance structure is defined via unitary invariant functions", IEEE Trans. Signal Process., 61(20), pp. 4949-4961 (2013).

29. Haykin, S., Kalman Filtering and Neural Networks, Wiley, New York (2001).
30. Kay, S.M., Fundamentals of Statistical Signal Processing, 2, Prentice Hall, New Jersey (1993).

\section{Biographies}

Mehdi Dorostgan received the MSc degree in Communication Engineering from Isfahan University of Technology (IUT), Iran in 2004. Currently, he is working on achieving the $\mathrm{PhD}$ degree in Communication Engineering at the Department of Electrical Engineering, Yazd University, Iran. His research interests include detection, estimation, and array signal processing.

Mohammad Reza Taban was born in Isfahan, Iran in 1968. He received the BSc degree in 1991 from Isfahan University of Technology (IUT), Isfahan, Iran, the MSc degree in 1993 from Tarbiat Modarres University, Tehran, Iran, and the PhD degree in 1998 from Isfahan University of Technology, all in Electrical Engineering. He joined the Yazd University Faculty, Yazd, Iran in 1999. Since 2016, he has been a Professor of Electrical and Computer Engineering at the Isfahan University of Technology, Isfahan, Iran. His main research interests are statistical signal processing, detection, and estimation. He has been in the scientific committee of the 10th international ISC conference (ISCISC2013) and the 2nd Iranian Conference on Avionics System (ICAS2015). Dr. Taban has published more than 90 technical papers in international and national journals and conferences. 